\title{
Desarrollo de Actitudes de Colaboración, Cooperación e Innovadoras en el Aula Basado en Proyectos
}

\author{
Development of collaboration, cooperation and innovation in the classroom based on \\ projects
}

Jorge Miguel Saldaña-Acosta

Recibido: 28/enero/2021

Aceptado: 8/abril/2021

Publicado: 16/abril/2021

\&aís

${ }^{1}$ México

\section{IIII Institución}

${ }^{1}$ Universidad Tecnológica Gral. Mariano Escobedo

\section{DCorreo Eletrónico \\ 13010jmsa@gmail.com}

\section{(iD ORCID}

${ }^{1}$ https://orcid.org/0000-0003-1484-1081

\section{Citar así: C APA / IEEE}

Saldaña-Acosta , J. (2021). Desarrollo de Actitudes de Colaboración, Cooperación e Innovadoras en el Aula Basado en Proyectos. Revista Tecnológica-Educativa Docentes 2.0, 11(1), 130-139. https://doi.org/10.37843/rted.v11i1.201

J. Saldaña-Acosta, "Desarrollo de Actitudes de Colaboración, Cooperación e Innovadoras en el Aula Basado en Proyectos", RTED, vol. 11, n. ${ }^{\circ} 1$, pp. 130139, abr. 2021.

\section{Resumen}

El aprendizaje basado en proyectos (ABP) es una herramienta fundamental para motivar a los estudiantes, brindándoles una oportunidad de involucrarse en su aprendizaje significativo, al sentirse como los verdaderos protagonistas. El objetivo de esta investigación fue promover el trabajo colaborativo, cooperativo e innovador para diseñar, desarrollar e implementar un prototipo el cuál permita gestionar un brazo robótico (autómata) empleando un microcontrolador Arduino. Se planteó el problema a resolver a un grupo de 16 estudiantes de $3^{\circ}$ grado de Ingeniería en Mecatrónica, UTSC; emplearon seis servomotores, tres estructuras de aluminio en $U$, una herramienta en forma de pinza, una placa Arduino uno, una fuente de energía, una interfaz USB, un ordenador, una base de madera donde serán sujetados los componentes; Ellos lograron estructurar un brazo robot de 6 grados de libertad, capaz de ejecutar los movimientos programados en el microcontrolador, además durante el desarrollo del proyecto se observó una notable mejora en su capacidad de trabajo en equipo, también su motivación por desarrollar el proyecto, así como el pensamiento crítico y plantear soluciones a los problemas enfrentados. El uso de robótica educativa en el aula a través del desarrollo de proyectos es una herramienta muy eficiente que conduce a los alumnos a desarrollar habilidades, fomentar el pensamiento crítico, llevar a un nivel de actuación real los diferentes conceptos o planteamientos teóricos vistos en clase.

Palabras clave: Innovación, proyectos, implementación.

\section{Abstract}

Project-based learning (PBL) is a fundamental tool to motivate students, allowing them to get involved in meaningful learning by feeling like the true protagonists. This research aimed to promote collaborative, cooperative, and innovative work to design, develop, and implement a prototype that allows managing a robotic arm (automaton) using an Arduino microcontroller. The presented problem to be solved was to a group of 16 students of the 3rd grade of Mechatronics Engineering, UTSC. They used six servo motors, three aluminum U-shaped structures, a clamp-shaped tool, an Arduino Uno board, a power source, a USB interface, a computer, a wooden base where they will hold components. They managed to structure a robot arm with 6 degrees of freedom capable of executing the microcontroller's movements. The observed development of the notable project improvement in their ability to work as a team and motivation develops the project. As critical thinking and propose solutions to the problems faced. The use of educational robotics in the classroom through the development of projects is a very efficient tool that leads students to develop skills, promote critical thinking, take the different concepts or theoretical approaches seen in class to a fundamental level of action.

Keywords: Active learning, production, textual understanding, TIC. 


\section{Introducción}

El aprendizaje basado en proyectos (ABP) es una herramienta fundamental para motivar a los estudiantes, brindándoles una oportunidad de involucrarse en su aprendizaje significativo, al sentirse como los verdaderos protagonistas. $\mathrm{Su}$ función primordial es fortalecer el aprendizaje de habilidades además de contenidos, mediante una enseñanza socializada; mantenerlos motivados además de comprometidos con su tarea o actividad a desarrollar; para los estudiantes es una necesidad apremiante e imprescindible, ellos presentan diferentes estilos de aprendizaje, niveles de habilidades, conocimientos previos o antecedentes culturales. Al tomar un rol activo se favorece su motivación académica, los estudiantes aprenden haciendo, el aprendizaje se vuelve realmente significativo (EDUforics, 2017).

A partir de los años 90, el área robótica ha incursionado de manera importante en diversas áreas, en una gran cantidad de actividades del hombre (García, Castillo \& Escobar, 2012); pasando actualmente a formar parte de nuestra sociedad (Mubin et al., 2013). El campo de educación también representa una importante área de impacto donde se busca un desarrollo, además del crecimiento intelectual del estudiante a través del uso de robots (Cabello, 2017), iniciativa de algunos pedagogos para mejorar las condiciones dentro del aula para el aprendizaje significativo al unificar conocimientos de física, electrónica, mecánica e informática través de la creación de un robot por parte de los estudiantes, donde aprenderán haciendo además desarrollaran un pensamiento lógico.

Incorporar proyectos de robótica en las aulas de clase ha mostrado ser una herramienta muy eficiente para desarrollar las habilidades de creatividad, el trabajo en equipo, de experimentar, además de lograr un aprendizaje a partir de reconocer el error; estas variables pueden impactar positivamente en los resultados académicos del alumno. Sin embargo, pocas escuelas incluyen dentro de su horario escolar esta herramienta por los elevados costos económicos, el desconocimiento de las herramientas o bien lo consideran como una actividad extracurricular, dejando de lado las posibilidades de motivación e integración, asociadas al uso de estas tecnologías en las aulas (Bravo \& Ferrero, 2012).

El objetivo de esta investigación fue promover el trabajo colaborativo, cooperativo e innovador en el aula mediante el uso del aprendizaje basado en proyectos, para diseñar, desarrollar e implementar un prototipo el cuál permita gestionar un brazo robótico (autómata), este llevará a cabo dos funciones específicas empleando un microcontrolador Arduino: 1) mover cada uno de los ejes del robot a cierta posición angular y b) Trasladar un objeto desde una posición hacia otra posición $\mathrm{B}$.

\section{Metodología}

Se planteó un problema a resolver a un grupo de 16 estudiantes de $3^{\circ}$ grado de Ingeniería en Mecatrónica, en la clase integradora I de la Universidad Tecnológica de Santa Catarina (UTSC); diseñar, desarrollar e implementar un prototipo el cuál permita gestionar un brazo robótico (autómata), el robot llevara a cabo 2 funciones específicas: 1) mover cada uno de los ejes del robot a cierta posición angular, 2) Trasladar un objeto desde una posición A hacia otra posición B; para ello emplearon seis servomotores, tres estructuras de aluminio en $\mathrm{U}$, una herramienta en forma de pinza, una placa Arduino uno, una fuente de energía, una interfaz USB, un ordenador, una base de madera para sujetarlos.

La primera tarea para realizar por el autómata fue mover cada uno de los ejes del robot a cierta posición angular. El robot cuenta con 6 servomotores para generar el movimiento mediante una señal PWM (Modulación de Ancho de Pulso) enviada a cada uno de los actuadores. El brazo robótico (ver Figura 1) será controlado a través de un microcontrolador Arduino, en el cual será cargado un algoritmo de control previamente programado en una computadora con el software necesario para especificarle los movimientos o tareas a ejecutar. 
previamente programada en el software Arduino 1.x.x. Los movimientos de posición angular programados y cargados al microcontrolador Arduino para su primera tarea están cargados en el código siguiente: \#include $<$ Servo.h $>/ /$ LIBRERÍA DE ARDUINO

\section{\#include $<$ Servo.h $>/ /$ LIBRERIA DE ARDUINO}

Servo myservo1;

Servo myservo2;

Servo myservo3;

Servo myservo4;

Servo myservo5;

Servo myservo6;

int pausa=2000,

void setup()

\{

myservol.attach(2);

myservo2.attach(3).

myservo3.attach(4);

myservo4.attach(5);

myservo5.attach(6);

myservo6.attach(7)

\}

void loop()

\{

myservol.write(0);

delay(pausa);

myservol.write(180);

delay(pausa);

myservo2.write (0);

delay (pausa);

myservo2.write(175);

delay (pausa);

myservo3.write (85).

delay(pausa);

myservo3.write(160);

delay(pausa);

myservo4.write(90);

delay(pausa);

myservo4.write(15);

delay(pausa);

myservo5.write(90).

delay(pausa);

myservo5.write(90);

delay (pausa);

myservo6.write(90);

delay(pausa);

myservo6.write(170);

delay(pausa);

myservo6.write(90);

delay(pausa);

myservo6.write(10);

delay(pausa);

\}

El robot tiene programada en el microcontrolador Arduino una segunda tarea por realizar; es trasladar un objeto de un punto $\mathrm{A}$ hacia un punto B. El código para este programa respecto al anterior respecto a de la primera tarea solo cambia a partir del void loop (), el cual es el siguiente:

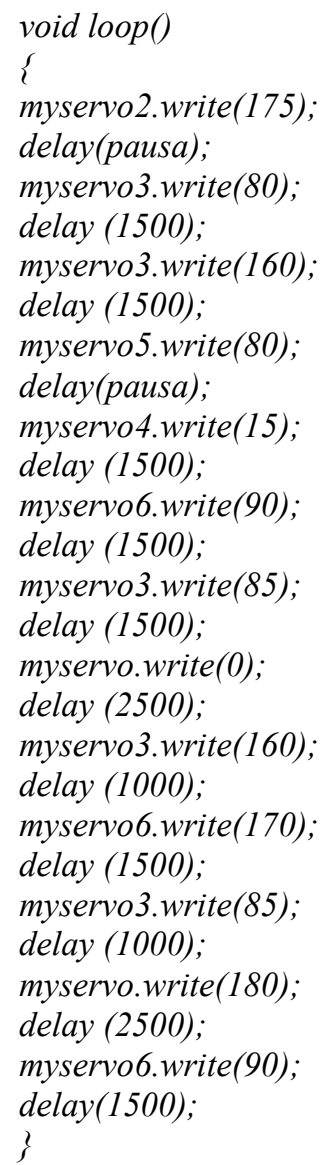

El impacto por desarrollar el proyecto de robótica en el aula sobre cuatro aspectos en los alumnos (trabajo en equipo, motivación, pensamiento crítico e innovación) se evaluó a través de un cuestionario sobre su apreciación en las actividades desarrolladas con respecto a estos parámetros con la siguiente valoración de 1 a 5 (1 Nada; 2 Muy Poco; 3 Poco; 4 Moderado; 5 Mucho). Se calculó el porcentaje de respuesta a cada parámetro por la población estudiada y la desviación estándar del nivel de impacto reportado.

Primera tarea del brazo. El robot realizo los movimientos de posición angular programados y cargados al microcontrolador Arduino para su primera tarea, se muestran en la Figura 6. El código utilizado para programar esta primera tarea es el siguiente \#include $<$ Servo.h $>$ //LIBRERÍA DE ARDUINO 


\section{Figura 6}

Movimientos de brazo robótico
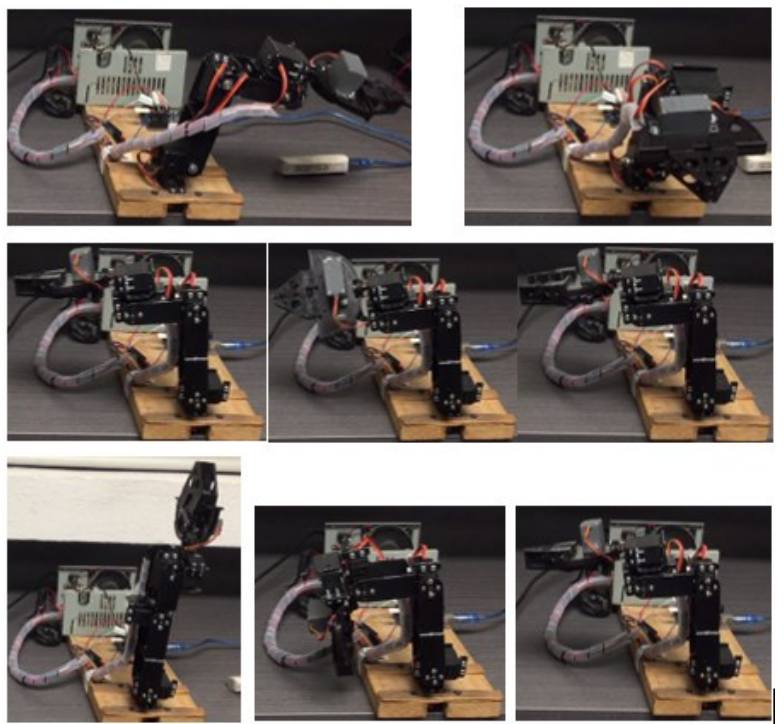

Nota. Vista de los diferentes movimientos angulares programados, elaboración propia (2020).

El brazo desarrollado y programado por los estudiantes, realizó apropiadamente su segunda tarea; trasladar un objeto de un punto a otro, ello se observa en la Figura 7 lo cual se programó, y cargo al microcontrolador Arduino.

\section{Figura 7}

Traslado de objeto de un punto a otro

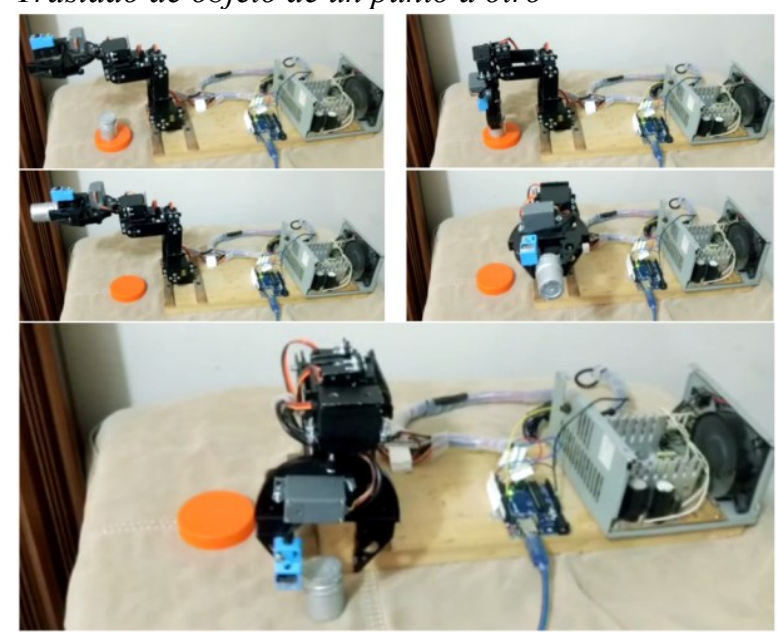

Nota. Traslado de un objeto de un punto A hacia otro punto B por el robot, elaboración propia (2020).

\section{Resultados}

Este enfoque pedagógico surgió a finales del siglo XIX además de a principios del siglo $\mathrm{XX}$ en USA, como en Europa por una necesidad de cambio para mejorar el proceso de aprendizaje del alumno. El conocimiento se adquiere de forma activa; el docente ayuda, guía al alumno a construir su conocimiento además de desarrollar habilidades, planteando problemas o proyectos para los alumnos; donde se pregunten, exploren, investiguen durante el proceso (Hidalgo-Morillo, 2017).

La incorporación de Arduino como recurso educativo de código abierto a nivel mundial ha creado diferentes tendencias de pensamiento con ideas encontradas. Ruiz-Corres (2016) comentado por Davis (2014) afirma sobre el uso de una plataforma Arduino con alumnos sin nociones previas de programación o electrónica, es una herramienta muy adecuada por ser real, no virtual, le permite al alumno trabajar con sensores además de actuadores, para desarrollar tareas o procesos específicos con equipos de bajo costo. Según Davis (2014) como herramienta de código abierto Arduino ha propiciado el surgimiento de múltiples plataformas de comunicación, soporte, foro o trabajo colaborativo; ello brinda a los alumnos la oportunidad de trabajar con cierta independencia lo que lo que le conduce a autoformarse de manera efectiva a través de propuestas de trabajo bajo el modelo de Aprendizaje Basado en Proyectos.

La robótica como ciencia surgió en los años 60; actualmente es un concepto de dominio público de gran interés en las comunidades educativas. En sus inicios esta ciencia era solo conocida por expertos, técnicos e ingenieros, por carecer de muchos conocimientos sobre esta área, ni tener el tipo de tecnología necesaria para aprovechar sus posibilidades. Hoy en día los avances en tecnología en campos de electrónica e informática ya están al alcance de todos. Toda sociedad actual se encuentra inmersa en una revolución tecnológica producto del desarrollo del transmisor semiconductor en 1951; este acontecimiento ha provocado cambios radicales como trascendentales en los ámbitos sociales, económicos o políticos con un impacto mundial; se le ha incorporado en muchos campos de ciencia, demostrando mucho potencial en nuevas aplicaciones; es también parte importante de nuestra vida diaria, donde todos empiezan a depender de ella.

Esta tecnología multidisciplinaria incorpora muchas ciencias en una única estructura, en donde los estudiantes pueden 
planear nuevos retos, les permite incorporar una competencia sana donde esta enseñanza se transforma en una diversión educativa. El objetivo de introducir robótica en el aula va más allá de simplemente introducir a los estudiantes de manera fáctica en los procesos productivos actuales, sino también pretende desarrollar habilidades motoras, sociales o de trabajo en equipo, reforzando el conocimiento en las demás áreas de conocimiento.

En el trabajo de robótica el estudiante enfrenta situaciones concretas donde se requieren soluciones prácticas además de una clase teórica para conducirle a un proceso de autoaprendizaje, de esta forma se potencia su representación de los conocimientos construidos, al permitir una interpretación del estudiante con los materiales, en resolución de problemas de manera colaborativa.

Al incorporar el área de robótica educativa en los centros escolares se pretende fortalecer las competencias a desarrollar por los estudiantes en sus diferentes niveles educativos, ello favorece su aprendizaje, estableciendo así las bases para innovar (ver figura 8). Con los avances tecnológicos logrados, además del desarrollo de habilidades informáticas en los estudiantes ha impulsado a esta área de robótica a convertirse en parte de una educación elemental en México (Valencia (2015)).

El planteamiento de una estrategia para resolver el problema planteado de construcción del robot impulsa a los alumnos a desarrollar su creatividad, desde proponer soluciones hasta evaluarlas mediante experimentación con el robot. El compartir estas experiencias, negociar además de manejar los recursos fortalece sus habilidades en el orden individual, las relaciones interpersonales de quienes participan en el desarrollo del robot. Así mismo el poner en operación el mecanismo robótico estimula su capacidad de análisis y crítica constructiva.

Al ser un hardware de código abierto Arduino ha permitido a otros fabricantes ofrecer placas con características idénticas con misma arquitectura (Cuartielles, 2012), además muy económicas ( $<3$ euros), ello motiva a los alumnos a comprar sus propios equipos e iniciar retos personales incrementando su conocimiento.

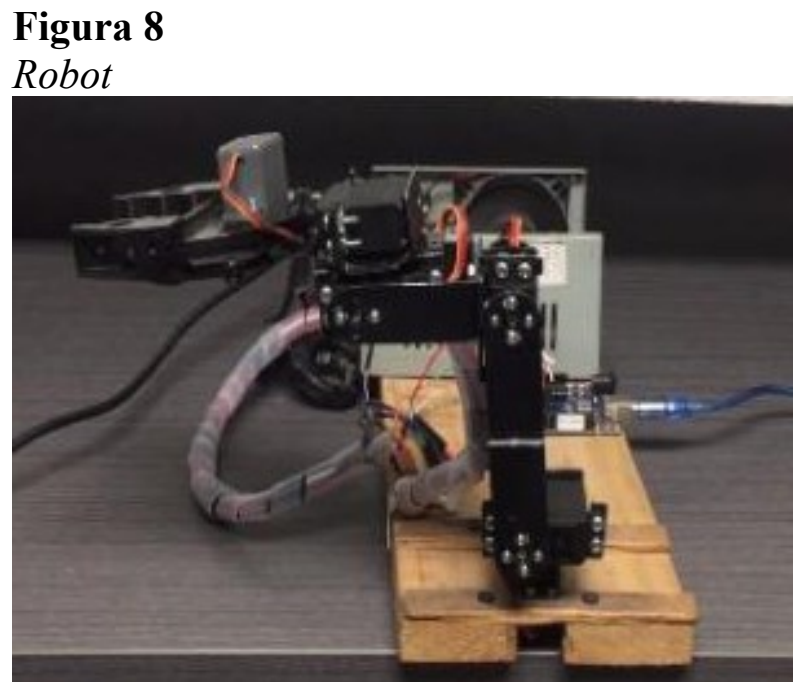

Nota. Vista lateral donde se muestran el antebrazo, el codo, el brazo y la pinza del robot, elaboración propia (2020).

Las actividades educativas con Arduino permiten ser diseñadas para trabajar no solamente las áreas de programación o electrónica sino conceptos relacionados con otras áreas del saber (matemáticas, física, ciencias de los materiales, etc.) así como del saber ser (trabajo en equipo, ayuda a los demás, trabajo autónomo) (De la Riva, 2015).

Los estudiantes lograron construir un brazo robótico con 6 grados de libertad, el robot fue capaz de ejecutar las tareas programadas al inicio del proyecto fue capaz de efectuar las tareas programadas, dentro de un ambiente de trabajo definido, consta de una base, antebrazo, codo, brazo, además tendrá una herramienta en forma de pinza al final de su muñeca para sujetar diversos objetos (ver Figura 9).

Los resultados obtenidos sobre el impacto registrado en los estudiantes por el desarrollo del proyecto planteado se determinó a partir de las respuestas obtenidas en los cuestionarios aplicados a los alumnos con respecto a su interés por desarrollar el proyecto, su capacidad de organización, coordinación para trabajar en conjunto, así como las habilidades mostradas en cuanto a los procesos de programación a través de pruebas de acierto y error, ver Gráfica 1.

La gráfica 1 muestra los niveles de impacto generados al desarrollar el proyecto de construcción de un robot por parte de los alumnos, estos resultados concuerdan con lo expresado por Bravo \& Ferrero (2012) \& y por Caballero (2017). 


\section{Gráfica 1}

Respuesta de los alumnos frente a la robótica

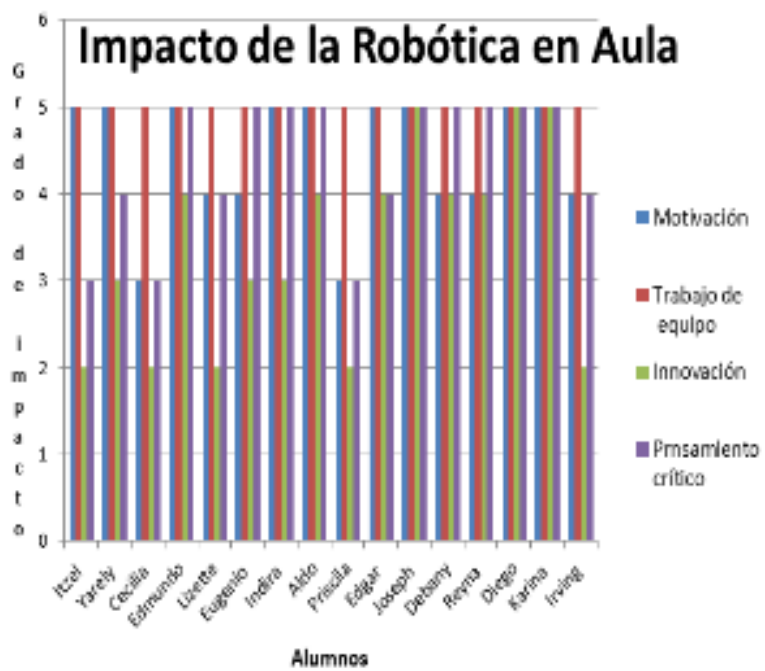

Nota. Nivel de impacto sobre el trabajo en equipo, pensamiento crítico, motivación e innovación por los alumnos de $3^{\circ}$ grado al desarrollar el proyecto asignado, elaboración propia (2020).

Una mayoría de los alumnos mejoraron su interés por los conceptos o actividades de aplicación práctica de sus conocimientos; mejoró su nivel de pensamiento crítico, mucho para el $56 \%$, moderado para un $25 \%$ y poco en un $19 \%$; en este parámetro se encontró una desviación estándar de 3.83 (ver gráfica 4); el desarrollo de su capacidad de innovación mostró una variabilidad menor, su desviación estándar fue de solo 1.15 , con valores de mucho en un $19 \%$, moderado en $31 \%$, poco $19 \%$ y muy poco $31 \%$, (ver gráfica 3); en cuanto a su motivación se observó un importante desarrollo de esta cualidad, sus valores fueron: mucho en un $56 \%$, moderado en $31 \%$, poco $13 \%$, su desviación estándar fue de 3.83 (ver gráfica 2).

\section{Gráfica 2}

Impacto sobre la motivación

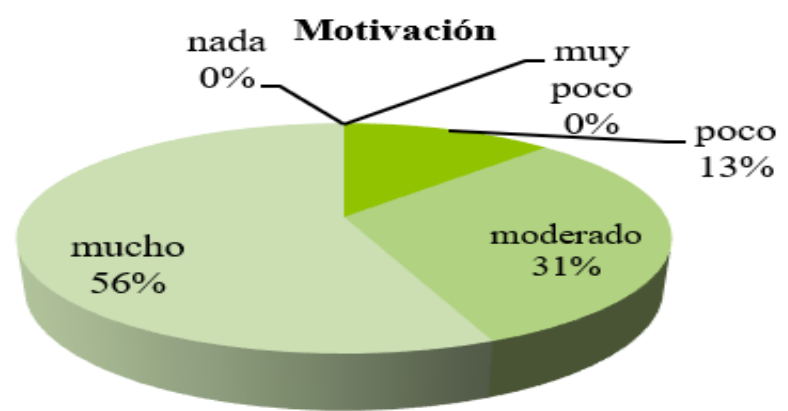

Nota. Porcentaje de respuesta por los estudiantes sobre su motivación al desarrollar el proyecto, elaboración propia (2020).

\section{Gráfica 3}

Impacto sobre la Innovación

\section{Innovación}

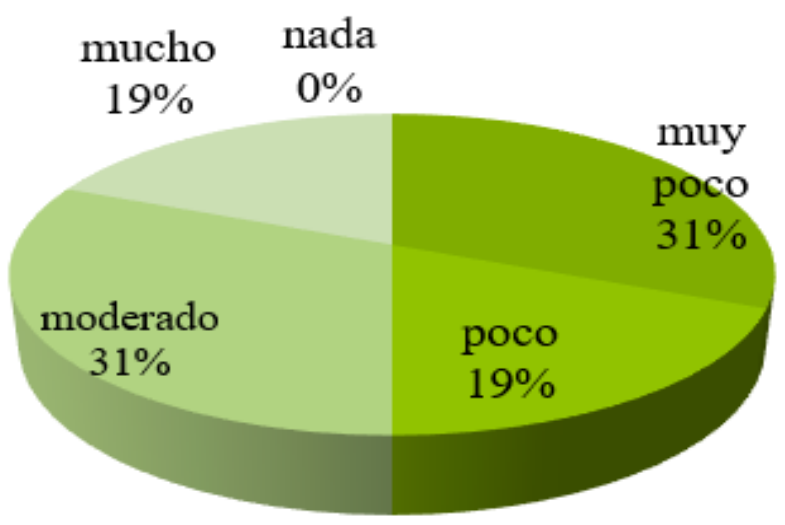

Nota. Respuesta de los alumnos sobre su innovación al desarrollar el proyecto, elaboración propia (2020).

\section{Gráfica 4}

Impacto sobre el pensamiento crítico

\section{Pensamiento crítico}

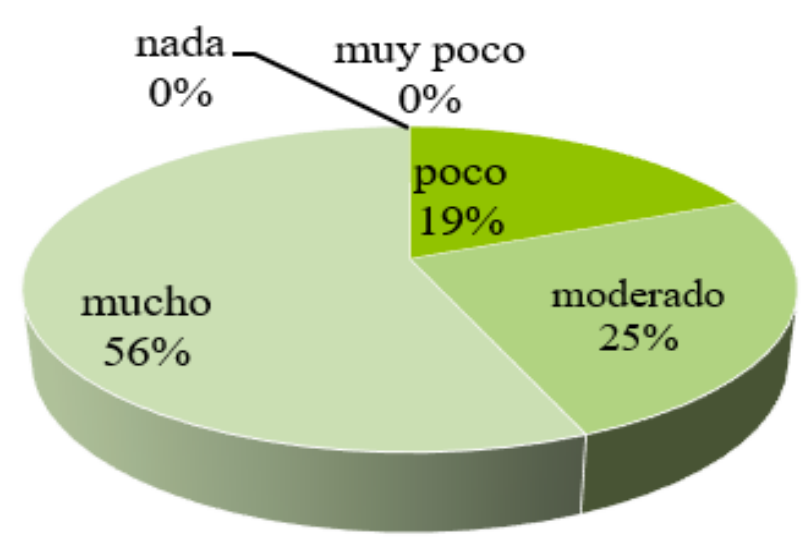

Nota. Porcentaje de Respuesta del impacto sobre los estudiantes en su pensamiento crítico al desarrollar el proyecto, elaboración propia (2020).

Primera Tarea para Realizar por el Brazo Robótico. El robot realizo los movimientos de posición angular programados y cargados al microcontrolador Arduino para su primera tarea, se muestran en la figura 9. El código utilizado para programar esta primera tarea es el siguiente \#include $<$ Servo.h $>$ //LIBRERÍA DE ARDUINO 


\section{Figura 9}

\section{Movimientos de brazo robótico}
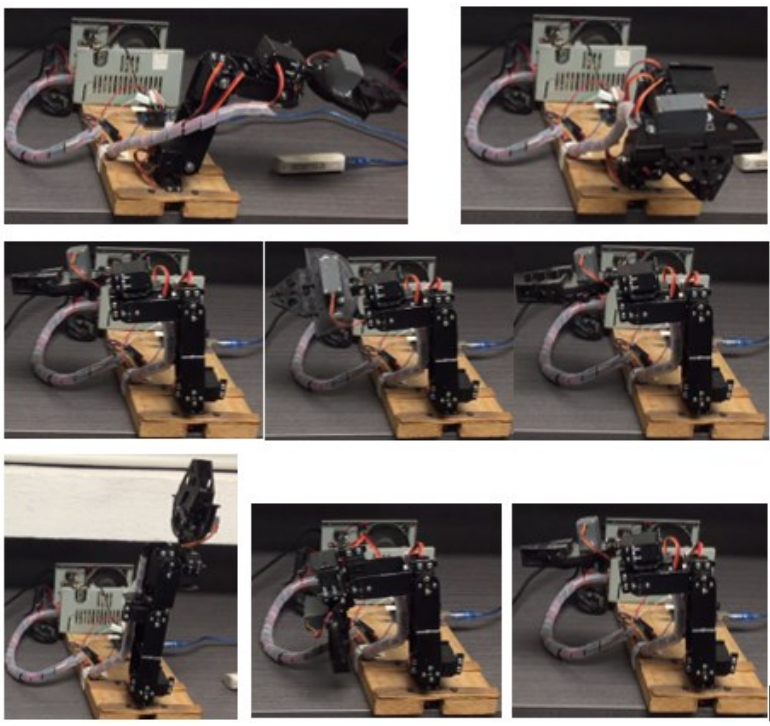

Nota. Vista de los diferentes movimientos angulares programados, elaboración propia (2020).

Segunda Tarea para Realizar por el Brazo Robótico. El brazo desarrollado, programado por los estudiantes, realizó apropiadamente su segunda tarea; trasladar un objeto de un punto a otro, ello se observa en la figura 10 lo cual se programó, y cargo al microcontrolador Arduino.

\section{Figura 10}

Traslado de objeto de un punto a otro

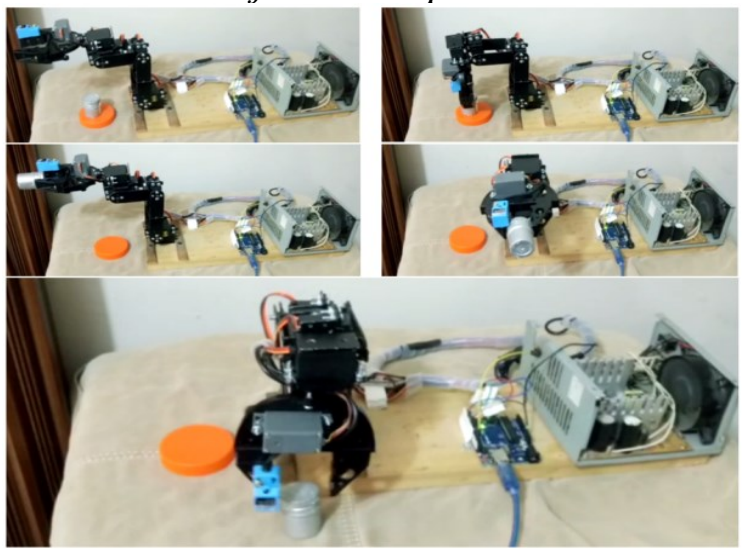

Nota. Traslado de un objeto de un punto A hacia otro punto B por el robot, elaboración propia (2020).

\section{Conclusiones}

La aplicación de la técnica educativa aprendizaje basado en proyectos a través del desarrollo del proyecto de construcción de un robot, fue muy eficaz al promover el trabajo en equipo e interés total por realizar el proyecto $(100 \%)$ en los estudiantes; con respecto a la habilidad de innovación se encontró una mayor dispersión en los resultados con un $31 \%$ de respuesta moderada; y en cuanto al pensamiento crítico y motivación se encontró un $56 \%$ para mucho como respuesta.

El diseño de robots involucra claramente una actividad cognitiva en los estudiantes, a través de una abstracción del problema para buscar soluciones, contribuyendo al desarrollo del pensamiento crítico además de innovar a nivel individual como colectivo. La elaboración e implementación de un robot integra una serie de actividades interdisciplinarias permiten reforzar los conceptos adquiridos en el aula, así como para asimilar nuevos conceptos de diversas áreas del conocimiento; matemáticas, física, ingeniería, biología, electrónica o programación entre otras.

Nuestros resultados están de acuerdo con los reportes de Davis \& Mubin (2014), Stevens, Shahid, Al Mahmudy \& Dong (2013) en cuanto al uso de robótica educativa como una muy buena herramienta para desarrollar habilidades, fomentar el pensamiento crítico e introducir en el área de la metodología científica al alumno, llevar a un nivel de actuación real los diferentes conceptos o planteamientos teóricos vistos en clase.

Educadores como Mubin, Stevens, Shahid, Al Mahmudy \& Dong (2013) resaltan el carácter motivador de herramientas como Arduino; esta herramienta busca una proactividad del alumno con diversas actividades, desde el diseño conceptual de un dispositivo hasta programar los propios controladores o su cableado e interconexión con sensores y actuadores. Ello resulta muy motivante para los estudiantes donde desarrollan lazos de pertinencia e identidad con el trabajo en desarrollo.

Este modelo responde de manera más eficaz a las necesidades del mundo del trabajo, al contexto social así como a las propias características de los estudiantes; representa una alternativa eficaz al mejorar la calidad del aprendizaje de los estudiantes, les permite trabajar en forma multidisciplinaria compartiendo metodologías para enseñar a aprender a través de estrategias profundas donde se crea, transfiere y produce abstracción de los contenidos, el trabajo autónomo colaborativo, promueve el aprendizaje significativo. El alumno 
es responsable de su aprendizaje. Nuestros resultados concuerdan con lo encontrado por Sánchez \& Ramis (2004) \& Sanmartí, (2009) en cuanto a su motivación, su aprendizaje significativo o su capacidad de trabajar en forma colaborativa.

\section{Referencias}

Arduino (2017). What is Arduino? hppt://www.arduino.cc/en/Guide/Introduction

Barrera- Lombana, N. (2015). Uso de la robótica educativa como estrategia didáctica en el aula. Praxis \& Saber, 6(11), 215 $-234$

Berkeley, M. (2017). Conectando el aprendizaje basado en proyectos al mundo real. https://www.gettingsmart.com/2017/03/connectingproject-based-learning-real-world/

Bravo, F.A., \& Ferrero, A. (2010). La robótica como un recurso para facilitar el aprendizaje y desarrollo de competencias generales. TESI en Teoría de la Educación. Educación y Cultura en la Sociedad de la Información, 13 (2), 120 136.1 hppt://campus.usal.es/" revistas_trabajo/index.php/revistatesi/article/view/9002/ 9247

Cabello, S., \& Carrera, X. (2017). Diseño y validación de un cuestionario para conocer las actitudes y creencias del profesorado de educación infantil y primaria sobre la introducción de la robótica educativa en el aula. EDUTEC, en Revista Electrónica de Tecnología Educativa, 60. hppt://www.edutec.es/revista

Cabello, J.A., Villapando, J.A., García, D., Bravo, J.A., Ortega, J.M., Álvarez, V.A., Cascajo, J.I., García, M., SanchezFerragut, R., \& Murillo, F. (2011). Unidad didáctica con placa ARDUINO hppt://educacionadistancia.juntadeandalicia.es/profesora do/pluginfile.php/2881/mod_resouce/content/1/Unidade s_Didácticas_Propuestas.pdf

Cuartilles, D., (2012). Arduino tecnologías abiertas y educación. [Adigital]. Archivo de video] youtube.com/watch?v=GGTH6hLCjTo

De la Riva, N. (2015). Arduino, La educación y la robótica. [Alcabot www.youtube.com/watch?v=roZbB4Top8

García, M.N., Castillo, L.F., \& Escobar A.J. (2012). Plataforma robótica educativa "ROBI" en Revista Colombiana de Tecnologías de Avanzada, 19(1), 140 - 144. hppt://www.unipamplona.edu.co/unipamplona/portalIG/ home_40/recursos/04_v19_24/revista_19/09022012/21. pdf

García, I. (2012). Aprendizaje basado en Problemas con ARDUINO. Trabajo final de Máster. Universidad de la Rioja hppt://biblioteca.unirioja.es/tfe_e/TFE000162.pdf

Gómez-Moreno C., Castillo-Solís A., \& Gómez-Meoño, A. (2015). Arduino como una herramienta para mejorar el proceso de enseñanza - aprendizaje de las ciencias, tecnologías e ingenierías en la Universidad Politécnica de Tapachula. Revista QUID, (24), 13 - 20.

Hidalgo-Murillo, B. (2017). Aprender a Desaprender [Blog]. Dinámica Basada en un Paisaje de Aprendizaje. https://aprenderdesaprendiendoblog.wordpress.com/201 $7 / 04 /$

Industrial Shields (2016). Industrial Shields-Controlador PLC basado en ARDUINO. www.Industrialshields.com

Jiménez-Castro Maynor y Cerdas-González Rosa Julia (2014, 12 14 de Noviembre). La robótica educativa como agente promotor del estudio por la ciencia y la tecnología en la región atlántica de Costa Rica. [Ponencia] Congreso Iberoamericano de Ciencia, Tecnología, Innovación y Educación. Argentina ISBN: 978-84-7666-210-6 Artículo https://www.oei.es/historico/congreso2014/contenedor.p $\mathrm{hp}$ ?ref=memorias

López- González, L., \& De-Pro-Bueno, A. (2017). Ideas del alumno sobre robótica y programación en $3^{\circ}$ de la ESO. [Ponencia] $X$ congreso nacional sobre investigación en didáctica de las ciencias. España, en Enseñanza de las ciencias $N^{\circ}$ Extraordinario (2017) 1261 - 1266.

Melendero, M. (2005). La Globalización de la educación. Ediciones Universidad de Salamanca, 3151, 185 - 208

Mubin, O., Stevens, C.J., Shahid, S., Al Mahmud, A., \& Dong, J. (2013). A review of the applicability of robots in education. Technology for Educcation and Learning, 17. hppt://roila.org/wp-content/uploads/2013/07/2090015.pdf

Monsalves-González, S. (2011). Estudio sobre la utilidad de la robótica educativa desde la perspectiva del docente. Revista de Pedagogía, 32 (90), 81 - 117.

Pereyra-Baz, M. A. (2015). 7 Elementos esenciales del ABP. https://cedec.intef.es/7-elementos-esenciales-del-abp/

Pittí-Patiño, K., Belén-Curto, D., Vidal-Morena, R., \& Rodríguez-Conde, J. (2014). Uso de la Robótica como Herramienta de Aprendizaje en Iberoamérica y España. VAEP - RITA, 2 (1), $41-48$

Rodríguez-Pérez, I., \& Madrigal-Arroyo, A. (2016). Rendimiento Académico y Estrategias de Aprendizaje. Revista de Docencia e Investigación Educativa, 2 (6), 26 - 34

Ruiz-Corres, D. (2016). Estudio sobre la implementación de la herramienta ARDUINO en centro de Formación Profesional. Trabajo final de Máster. Tecnologías de la Información y Comunicación (TIC) Universidad Internacional de la Rioja (UIR). https://reunir.unir.net/bitstream/handle/123456789/4540/ RUIZ\%20CORRES\%2C\%20DANIEL.pdf?sequence=1 \&isAllowed $=\mathrm{y}$

Ruiz-Gutierrez, J.M. (2012). Utilización de $S_{4 A}$ (Scratch) más la tarjeta Arduino en un ambiente de programación gráfico orientado a la educación. http://s4a.cat/downloads/s4amanual.pdf

Schwab, K. (2010). La cuarta Revolución Industrial. Editorial Debate. hppt://www.hackvandedm.nl/blog/?p=762 
Willging, P. A., Astudillo, G. J., Castro, L., Bast, S., Occelli M., \& Distel Juan. (2017). Educación con Tecnologías: la Robótica educativa Aplicada para el aprendizaje de la programación. [Conferencia]. XIX Workshop de Investigadores en Ciencias de la Computación, Argentina 